\section{EDUCATION}

Research, Inovertion and Solutions on-line $e^{6}$
PSYCHOLOGY

$1+D+i$
Electronic Journal of Research

in Educational Psychology

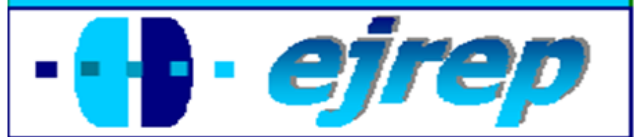

\title{
Control de la acción y disposición a la esperanza: Un estudio de su incidencia en la autorregulación del aprendizaje
}

\section{Georgia Papantoniou ${ }^{1}$, Despina Moraitou ${ }^{2}$, Effie Katsadima ${ }^{1}$, Magda Dinou ${ }^{1}$}

${ }^{1}$ Departamento de Educación Infantil, Universidad de Ioánnina

${ }^{2}$ Facultad de Psicología, Universidad Aristóteles de Tesalónica

Grecia

Correspondencia: Georgia Papantoniou. Department of Early Childhood Education, School of Education, University of Ioannina, 45110 Ioannina, Greece. E-mail: gpapanto@uoi.gr

(C) Education \& Psychology $\mathrm{I}+\mathrm{D}+\mathrm{i}$ and Editorial EOS (Spain) 


\section{Resumen}

Introducción. En el presente estudio se examina la incidencia del control de acción (i.e., separación, iniciativa, y persistencia) y la disposición a la esperanza (i.e., pensamiento orientado a caminos, y pensamiento orientado a la agencia) en el uso de estrategias de aprendizaje autorregulado (i.e., cognitivas, metacognitivas, y gestión de recursos) y en el logro académico.

Método. Un total de 275 estudiantes de grado de la Facultad de Educación participaron en la investigación. Los datos se recogieron durante una asignatura de Psicología. Se invitó a los participantes a contestar la Action Control Scale [Escala de Control de Acción] (ACS-90) y la Adult Dispositional Hope Scale [Escala para Adultos sobre Disposición a la Esperanza] (ADHS). También cumplimentaron las Learning Strategies Scales [Escalas de Estrategias de Aprendizaje] del MSLQ. Se utilizaron las calificaciones académicas como medida de logro académico.

Resultados. Los resultados de los análisis del camino muestran que los dos componentes de la disposición a la esperanza influyeron en el uso de casi todas las estrategias de aprendizaje. La separación y la iniciativa influían sobre todo en la gestión del tiempo y el entorno de estudio, y en la regulación del esfuerzo. La persistencia y el pensamiento orientado a caminos contribuyeron de forma independiente a la varianza del logro académico, mientras que la metacognición, y la gestión del tiempo y del entorno de estudio mediaron los efectos de separación, iniciativa y control del pensamiento.

Discusión y Conclusión. En conclusión, la contribución principal del presente trabajo es la identificación del impacto que pueden tener características de rasgo, como el control de la acción y la disposición a la esperanza, en el uso de estrategias de aprendizaje autorregulado y en el logro académico.

Palabras Clave: control de acción, logro académico, disposición a la esperanza, estrategias de autorregulación de aprendizaje.

Recibido: 17/12/09 Aceptación Inicial: 23/12/09 Aceptación Definitiva: 20/01/10 


\begin{abstract}
Introduction. The present study examined the effect of action control (i.e., disengagement, initiative, and persistence) and dispositional hope (i.e., pathways thought, and agency thinking) on self-regulated learning strategy use (i.e., cognitive, metacognitive, and resource management) and course achievement.
\end{abstract}

Method. A total of 275 undergraduate students at School of Education participated in the study. Data were collected during a psychology course. The participants were asked to respond to the Action Control Scale (ACS-90) and the Adult Dispositional Hope Scale (ADHS). They also completed the Learning Strategies Scales of the MSLQ. Examination grades were used as the measure of course achievement.

Results. The results from path analysis suggest that the two components of dispositional hope influenced the use of almost all the learning strategies. Disengagement and initiative were found to influence mainly time and study environment management and effort regulation. Persistence and pathways thought made an independent contribution to the variance of course achievement, whereas metacognition and time and study environment management mediated the effects of disengagement, initiative and agency thinking.

Discussion and Conclusion: In conclusion, the main contribution of the present study is the identification of the impact that trait-like characteristics, such as action control and dispositional hope, might have on university students' self-regulated learning strategy use and course achievement.

Keywords: action control; course achievement; dispositional hope; self-regulated learning strategies.

Received: 12/17/09 Initial Acceptance: 12/23/09 Definitive Acceptance: 01/20/10 


\section{Introducción}

En los últimos 20 años, se ha conseguido un progreso considerable en cuanto al concepto del aprendizaje autorregulado, sus componentes y su funcionamiento (Pintrich, 2000; ver Torrano y González, 2004). El proceso de autorregulación hace referencia a la supervisión y el control de la persona sobre su rendimiento, cognición, y afecto, así como su entorno, para lograr sus metas (Efklides, Niemivirta, y Yamauchi, 2002; Pintrich, 2000). La importancia del aprendizaje autorregulado está muy establecida, particularmente en los alumnos de secundaria o universitarios, ya que se ha mostrado que los atributos de los aprendices autorregulados están relacionados positivamente con el logro académico y con la calidad del aprendizaje y el rendimiento (Boekaerts, 1997; Pintrich y DeGroot, 1990; Schunk y Zimmerman, 1994).

En general, hay dos ideas ampliamente promocionadas en la investigación del aprendizaje autorregulado. Primero, la idea de que la autorregulación incorpora diferentes sistemas y procesos que monitorizan y controlan el comportamiento, tales como la atención, la metacognición, la motivación, la emoción y el control de acción (p.ej., Kuhl y Fuhrmann, 1998; Zimmerman, 1999). Segundo, la idea de que es importante que los investigadores de la autorregulación estudien el uso real de las estrategias de aprendizaje autorregulado en entornos académicos (Zimmerman, 1999). Bajo el marco de la segunda idea, la autorregulación en el entorno académico se concibe como una serie de habilidades que pueden desarrollarse, más que ser algo inalterable o enraizada genéticamente (Pintrich, 1995). Como resultado, varios aspectos del aprendizaje autorregulado, en su trato conceptual, se han considerado variables situacionales, dependientes del contexto, mientras que se le ha dado poca atención a la conexión entre el aprendizaje autorregulado y las características individuales de tipo rasgo (Bartels, Magun-Jackson, y Kemp, 2009; Bidjerano y Dai, 2007; Hong y O’Neil, 2001). Aunque las predisposiciones individuales para desarrollar y ejercer habilidades autorreguladas puede ser una fuente menos reconocida del aprendizaje autorregulado, en su teoría de control de acción de la autorregulación, Kuhl (1985) identificó la orientación acción-estado como una perspectiva de diferencias individuales a la hora de ver diferencias estilísticas en la autorregulación de acción.

En cuanto al concepto del aprendizaje autorregulado, una de las preguntas sin contestar es el grado en que éste queda asociado con disposiciones de personalidad estables. Por lo tanto, este trabajo trata un conjunto de estrategias de autorregulación de aprendizaje que fueron identificadas en investigaciones previas como elementos básicos de los procesos de autorregulación de aprendizaje, y su relación con dos disposiciones relacionadas con metas, a saber, el control de acción y la disposición a la esperanza. 


\section{Estrategias de autorregulación de aprendizaje}

Los investigadores han identificado varios procesos autorreguladores que explican la complejidad y variabilidad en los esfuerzos de los alumnos por aprender por sí mismos. El interés en este estudio es la autorregulación tal como la propone el modelo de Pintrich (1999), el que comprende tres categorías generales de estrategias: estrategias de aprendizaje cognitivo, estrategias de control metacognitivo o autorreguladoras, y estrategias de gestión de recursos tales como la gestión y control de su propio tiempo, esfuerzo, entorno de estudio, etc.

Las estrategias cognitivas (incluidas el ensayo, la elaboración, la organización, y el pensamiento crítico) son formas de las que los aprendices manipulan información en respuesta a las necesidades de la tarea, tales como escoger entre información principal e información trivial y centrar la atención (Pintrich, 1999; Pintrich y DeGroot, 1990). Las estrategias cognitivas pueden reflejar un nivel profundo de procesamiento o no (Pintrich, 1999). El ensayo, la estrategia más básica de aprendizaje para procesar la información, representa la repetición verbal del material con la meta de memorizarlo. La elaboración, una habilidad de aprendizaje de orden superior, se define operacionalmente como parafrasear y resumir. La organización incluye estrategias tales como el bosquejo, tomar apuntes y conectar diferentes aspectos del material estudiado. La estrategia de aprendizaje Pensamiento Crítico consiste en evaluaciones críticas de ideas y la aplicación del conocimiento a nuevas situaciones (Pintrich, Smith, Garcia, y McKeachie, 1991).

Las estrategias metacognitivas (es decir, planificación, supervisión y regulación) consideradas a menudo el aspecto principal de la autorregulación del aprendizaje, representan funciones ejecutivas diseñadas para evaluar y controlar el uso de las estrategias cognitivas (Pintrich et al., 1991). La planificación implica fijar metas y resultados educativos al igual que el análisis de tareas. Los aprendices autorregulados fijan resultados de aprendizaje o de rendimiento, y luego supervisan la eficacia de sus métodos de aprendizaje o estrategias y responden a sus evaluaciones. La supervisión es esencial para mejorar el aprendizaje. Ayuda a los alumnos a centrar su atención y a discriminar entre actuación eficaz e ineficaz, y revela estrategias de aprendizaje inadecuadas. También mejora la gestión del tiempo.

Las estrategias de Gestión de Recursos (el uso eficaz del tiempo y del entorno de estudio, regulación de esfuerzo, aprendizaje entre iguales, y la búsqueda de ayuda) buscan establecer condiciones que faciliten el aprendizaje (Pintrich et al., 1991). La Gestión del Entorno de Estudio requiere localizar un lugar que sea tranquilo y relativamente libre de distracciones visuales y auditivas y que permite al alumno concentrarse. La gestión del tiempo 
implica programar, planificar y gestionar el propio tiempo de estudio. La estrategia de Regulación de Esfuerzo es parecida en significado a la volición y puede verse como una estrategia de control de acción (Kuhl, 1985). En términos de la teoría moderna de la volición, esta refleja el compromiso del alumno con sus propias metas de estudio al dirigir y controlar su energía hacia ellas, y al tratar los reveses y fracasos en el proceso de aprendizaje, asignando más esfuerzo a las tareas desempeñadas sin éxito. En una situación académica, la regulación de esfuerzo puede usarse para construir destrezas de aprendizaje gradualmente y para ayudar a los alumnos a tratar con las muchas distracciones dentro y fuera de los centros educativos (Chen, 2002). El aprendizaje entre iguales es usar un grupo de estudio o amigos que ayuden a aprender (Pintrich et al., 1991). La Búsqueda de Ayuda se refiere a buscar ayuda de otros - iguales o docentes- al encontrar dificultades de aprendizaje (Pintrich et al., 1991). El buscar la ayuda de otros es una estrategia de aprendizaje preactiva y autorregulada, que puede proveer el fundamento para el logro autónomo.

En resumen, se ha mostrado que los atributos de los aprendices autorregulados, tales como la organización y la transformación de información, la supervisión de la eficacia de sus métodos de aprendizaje, y la utilización de recursos del entorno, parecen estar relacionados positivamente con y directamente implicados en el rendimiento, y la buena adaptación escolar.

\section{Control de acción, estrategias de autorregulación de aprendizaje y el rendimiento}

La orientación acción-estado es un constructo de personalidad global que trata las diferencias individuales en la capacidad para regular las emociones, cogniciones y comportamientos para llevar a cabo acciones intencionadas (e.g., Kuhl, 1994b). El constructo de orientación acción-estado se mide en un continuo con dos extremos: la orientación a la acción y la orientación al estado.

Debido a su enfoque en los asuntos dinámicos y procesuales de la lucha por metas, la orientación acción-estado puede ser útil para comprender por qué dos individuos con metas, conocimiento, y capacidad similares, y el mismo deseo de buena ejecución, no alcancen el mismo nivel de rendimiento. Aunque es probable que los individuos caigan en un continuo de orientación acción-estado, es útil contrastar los individuos mayormente orientados a la acción con aquellos más orientados al estado. Los individuos con una fuerte orientación a la acción son capaces de dedicar sus recursos cognitivos a la tarea en mano, lo cual les permite pasar oportunamente desde un estado de meta presente a algún estado deseado de meta futura. Estos individuos asignan su atención flexiblemente al propósito de ejecutar una tarea o al de obtener una meta. Las personas que son mayormente orientadas a la acción se caracterizan por una 
mayor eficacia en el rendimiento (Kuhl, 1994b) y por la capacidad de completar tareas después de pequeños fracasos o reveses. En cambio, los individuos con una mayor orientación al estado suelen tener pensamientos persistentes y reflexivos sobre metas o estados afectivos alternativos, lo cual reduce los recursos cognitivos disponibles para alcanzar la meta. Esta reducción de recursos disponibles afecta la capacidad de los individuos orientados al estado para poder iniciar actividades y continuar las tareas hasta finalizarlas, especialmente cuando las actividades son difíciles, no rutinarias, o ambas cosas (Brunstein y Olbrich, 1985; Kuhl, 1994b). Además de la noción general de orientación acción-estado, Kuhl (e.g., Kuhl y Beckmann, 1994) sugiere que hay tres aspectos o dimensiones distintos que pueden distinguirse: Preocupación (frente a la Separación), Vacilación (frente a la Iniciativa), y Volatilidad (frente a la Persistencia).

Dimensión Preocupación (frente a Separación). Esta dimensión, con los polos opuestos de preocupación frente a separación, indica el grado en el cual los individuos explícitamente procesan información relacionada con algún estado pasado, presente o futuro. El polo de esta dimensión orientado a la acción (la separación) se refiere a la capacidad de separarse de pensamientos sobre metas alternativas o eventos no deseados que puedan interferir en el progreso de la tarea en mano. En cambio, el polo orientado al estado está asociado con eficacia disminuida debido a la perseverancia de pensamientos relacionados con alguna experiencial desagradable (real o simulada), que a menudo involucra un fracaso (ver Diefendorff, Hall, Lord, y Strean, 2000; Kuhl, 1994a).

Dimensión de Vacilación (frente a Iniciativa). Esta dimensión, con los polos opuestos de la vacilación frente a la iniciativa, se refiere al grado en el cual los individuos tienen dificultad para iniciar actividades previstas que van dirigidas hacia una meta. Los individuos orientados a la acción en esta dimensión (polo de iniciativa) son capaces de ponerse a trabajar en las tareas fácilmente. Aunque tanto la dimensión de iniciativa como la de separación son similares en su enfoque hacia la capacidad de avanzar en las tareas y de escapar del procesamiento orientado al estado cuando es necesario, ambos tienen bases diferentes. Concretamente, la dimensión de separación tiene que ver con los pensamientos que le distraen y pueden interferir a la hora de iniciar la acción, mientras que la dimensión de iniciativa enfatiza la capacidad conductual para iniciar la acción (see Diefendorff et al., 2000; Kuhl, 1994a).

Dimensión de Volatilidad (frente a Persistencia). Esta dimensión con los polos opuestos de la volatilidad frente a la persistencia tiene que ver con la capacidad de permanecer en el modo orientado a la acción cuando es necesario. Se refiere al grado en el cual los individuos se distraen mientras trabajan en una tarea interesante o necesaria. Los individuos más orientados a la acción (polo de persistencia) son capaces de mantenerse 
eficazmente centrados en alguna intención hasta que finalizan la tarea, mientras que los individuos con mayor orientación al estado son fácilmente desviados de la tarea, perjudicando así su rendimiento global. Procesamiento orientado al estado y asociado a la dimensión de volatilidad puede deberse a una hiperactividad del sistema de inicio de acción (y, por lo tanto, una tendencia a iniciar nuevas tareas de manera inapropiada), en vez de a la poca actividad de este sistema que se ve en los polos orientados al estado en las dimensiones de preocupación y vacilación (ver Diefendorff et al., 2000; Kuhl, 1994a).

Por lo tanto, las tres dimensiones orientadas a la acción tienen que ver con diferentes facetas del proceso para alcanzar metas. Los individuos orientados a la acción se desconectan flexiblemente de asuntos irrelevantes (separación), inician de forma eficaz las acciones necesarias (iniciativa), y permanecen centrados hasta que se finalizan las tareas (persistencia).

Esta declaración lleva implícita la noción de que la orientación a la acción es más adaptiva que la orientación al estado durante el desempeño y la selección de las estrategias de resolución eficaces (p.ej., Brunstein y Olbrich, 1985; Kuhl, 1994b), aunque una revisión de la literatura (Kuhl y Fuhrmann, 1998) revela que la orientación al estado no es siempre disfuncional. Perry, Hladkyj, Pekrun, y Pelletier (2001) examinaron el control de acción usando solamente la subescala de Preocupación, la elaboración cognitiva y estrategias metacognitivas de supervisión propia con escalas adaptadas del MSLQ, la toma de apuntes con un solo ítem y el logro académico con la nota de final de curso. Ellos encontraron que los alumnos con una alta preocupación de fracaso tomaron más apuntes durante las clases y consiguieron mejores notas finales. El uso por parte de los alumnos de las estrategias de elaboración y estrategias metacognitivas no estaba correlacionado de forma significativa con la preocupación de fracasar. Bembenutty, Karabenick, McKeachie, y Lin (1998) examinaron el control de acción usando una escala basada en el componente de comportamiento de la escala más extensa de control de acción de Kuhl (1985). Ellos evaluaron también el rendimiento académico de los alumnos y su uso de estrategias de aprendizaje con una versión modificada del MSLQ. Se encontró que el control de acción estaba correlacionado positivamente con el uso de todas las estrategias cognitivas, metacognitivas y autorreguladoras, excepto las del pensamiento crítico, la atención y el aprendizaje entre iguales. Papantoniou (2002) examinó el efecto del control de acción sobre el uso de estrategias de aprendizaje y el rendimiento cognitivo. La orientación a la acción (separación, iniciativa y persistencia) se midió con la escala de Kuhl (1994b) de 36 ítems, la ACS-90. Ésta evaluó la autorregulación según dos categorías de estrategias: 1) estrategias profundas, y 2) técnicas de aprendizaje. Los análisis del camino mostraron que la iniciativa y la persistencia facilitan el rendimiento cognitivo (en la comprensión de textos y en la orientación espacial) a través de la regulación del uso de estrategias de aprendizaje por parte de los alumnos. 
Jaramillo y Spector (2004) examinaron el efecto del control de acción sobre el esfuerzo y el rendimiento académico. La orientación a la acción (separación, iniciativa y persistencia) se midió con la escala de 22 ítems de Diefendorff et al. (2000), la cual se basa en la escala de 36 ítems de Kuhl (1994b). De acuerdo con su modelo, la iniciativa y la persistencia están relacionadas positivamente con el esfuerzo lo cual es un antecedente importante del rendimiento académico. Diefendorff (2004) examinó el papel de la orientación acción-estado para predecir la motivación y el rendimiento en una tarea específica dentro del contexto académico. La orientación de acción (la separación y la iniciativa) se midió usando las respectivas subescalas de la escala de 22 ítems de Diefendorff et al. (2000). Los resultados de este trabajo mostraron que la separación y la iniciativa predicen el rendimiento independientemente de la orientación hacia la meta, la capacidad cognitiva, la autoeficacia y las metas auto impuestas. Roy, Vezeau, y Bouffard (2008) examinaron el control de acción usando sólo las subescalas de Preocupación y Vacilación. Ellos evaluaron la autorregulación según cuatro categorías de estrategias: 1) la planificación y la organización, 2) el esfuerzo por entender más, 3) la implicación, y 4) la perseverancia. Ellos encontraron que la preocupación de fracasar apenas está vinculada con la autorregulación. Roy, Vezeau, y Bouffard (2008) encontraron también que el uso de cada una de las estrategias autorreguladoras correlacionaba de forma negativa con la vacilación. En resumen, la reciente evidencia empírica muestra un patrón variado de relaciones del control de acción con las estrategias de autorregulación de aprendizaje y con las variables de rendimiento.

\section{La disposición a la esperanza y el rendimiento}

Al igual que el control de acción, la esperanza es bastante prometedor como constructo relacionado con las metas: una de las formas de gestionar las metas y adaptarse a los retos de la vida diaria es con la esperanza. La esperanza contribuye a la satisfacción y eon la longevidad, y por esta razón es identificada en la psicología positiva como una fortaleza humana (Danner, Snowdon, y Friesen, 2001; Kashdan, Pelham, Lang, Hoza, Jacob, Jennings, Blumenthal, y Gnagy, 2002).

Durante la última década, la esperanza se estableció firmemente como una estrategia de afrontamiento esencial en varios dominios, incluido el académico (ver Snyder, 2000). Aunque es frecuente el equiparar la esperanza con hacerse ilusiones, Snyder y sus compañeros (Snyder, Harris, Anderson, Holleran, Irving, Sigmon, Yoshinobu, Gibb, Langelle, y Harney, 1991) definen la esperanza con un significado preciso y operacional. En este trabajo, la esperanza se conceptualiza conforme a la teoría de Snyder et al. (1991; ver también Snyder, 2000) como una disposición dirigida a una meta, la cual consiste en de dos componentes interrelacionados pero distintos: (a) Waypower, o el pensamiento orientado a caminos, se 
refiere a la capacidad percibida de identificar caminos alternativos- es decir, estrategias cognitivas hacia las metas deseadas o valoradas- y también la capacidad de diálogo interno positivo sobre ser capaz de encontrar esos caminos, (b) la fuerza de voluntad, o pensamiento orientado a la agencia, refleja la dimensión motivadora de la esperanza. Se refiere a la capacidad percibida de ratificar diálogo interno sobre la capacidad de actuar (agencia). El pensamiento orientado a la agencia conlleva especial importancia cuando las personas encuentran impedimentos. En tales situaciones el pensamiento orientado a la agencia y el diálogo interno ayudan a la persona a dirigir la motivación necesaria hacia el mejor camino (Snyder, Rand, y Sigmon, 2002). En otras palabras, las personas con un alto nivel de esperanza tienen tanto los "caminos" como la "voluntad" para conseguir sus metas. De acuerdo con la teoría, el pensamiento orientados a caminos y el pensamiento orientado a la agencia trabajan conjuntamente; son "recíprocas, aditivas y están relacionados de forma positiva entre sí" (Snyder et al., 1991, p. 370). Esta dualidad de los componentes de la esperanza-agencia y caminos-es lo que distingue claramente la esperanza de la autoeficacia. La autoeficacia, la creencia en las propias aptitudes para organizar y llevar a cabo una línea de acción (Bandura, 1997), comparte cierta similitud con la agencia (fuerza de voluntad), como componente de esperanza, pero difiere de la esperanza al no incorporar el componente de los caminos.

Las investigaciones muestran que la esperanza tanto en su forma de rasgo como en la de estado, es un predictor eficaz de varios comportamientos relacionados con el rendimiento (Snyder, 2000). Curry, Snyder, Cook, Ruby, y Rehm (1997) encontraron que entre los atletas, esperanza de estado mediaba la relación entre esperanza de rasgo y el rendimiento atlético. Peterson, Gerhardt, y Rode (2006) examinaron y encontraron apoyo parcial para un modelo de relaciones entre esperanza de rasgo, esperanza de estado, orientación hacia la meta de aprendizaje, indicadores verbales, y la ejecución de tareas. En lo que se refiere a las variables de la esperanza, encontraron que una orientación hacia la meta de aprendizaje estaba relacionada de forma positiva y significativa con esperanza de rasgo y que la esperanza de rasgo estaba relacionada de forma positiva con la ejecución en una tarea de anagrama a través de la mediación de la variable esperanza de estado.

Aunque el pleno conocimiento del papel de la esperanza en la ejecución de tareas nos informaría sobre la relativa eficacia de la esperanza como un constructo que mejora el rendimiento, hasta donde sabemos, aún no se ha investigado adecuadamente el grado en el cual se relaciona la esperanza con la autorregulación del aprendizaje y el rendimiento evaluado por notas académicas. 


\section{Objetivo - Hipótesis}

Por consiguiente, el presente trabajo tiene como objetivo examinar la incidencia del control de acción (es decir, separación, iniciativa, y persistencia) y la disposición a la esperanza (es decir, pensamiento orientado a caminos, y pensamiento orientado a la agencia) en el uso de estrategias de autorregulación del aprendizaje (es decir, cognitivas, metacognitivas y gestión de recursos) y en el logro académico.

A partir de trabajos recientes que de forma general muestran que el control de acción puede estar correlacionado con las estrategias de autorregulación del aprendizaje, hacemos la hipótesis de que la orientación a la acción puede estar asociada con el uso de estrategias cognitivas, metacognitivas y autorreguladoras (Hipótesis 1). Específicamente, basándonos en los trabajos de Bembenutty et al. (1998), Jaramillo y Spector (2004), Papantoniou (2002), y Roy, Vezeau, y Bouffard (2008), hacemos la hipótesis de que la iniciativa y la persistencia pueden estar positivamente asociadas con el uso de estrategias de aprendizaje (Hipótesis 1a), mientras que, basándonos en los hallazgos de Perry et al. (2001), esperábamos asociaciones negativas entre la separación y las estrategias de autorregulación del aprendizaje (Hipótesis $1 b)$.

Aunque aún no se ha establecido la relación entre la disposición a la esperanza y habilidades específicas de autorregulación del aprendizaje, nos planteamos la hipótesis de que el concepto de la esperanza, como una capacidad percibida de poder (1) conceptualizar metas claramente, (2) desarrollar estrategias específicas para lograr las metas (pensamiento orientado a caminos), y (3) iniciar y mantener la motivación a la hora de usar estas estrategias (pensamiento orientado a la agencia) (Snyder, 2000; Snyder et al., 2002), puede estar asociado con el uso de habilidades de autorregulación del aprendizaje (Hipótesis 2).

Ya que las diferencias individuales de rasgos están más distantes del rendimiento que las diferencias individuales en el uso de estrategias de aprendizaje, nos planteamos la hipótesis de que las relaciones entre el control de acción y la disposición a la esperanza con el rendimiento evaluado con notas académicas deberían ser indirectas, a través de las habilidades de autorregulación del aprendizaje. Específicamente, debido a que varios trabajos (Bidjerano y Dai, 2007; Chen, 2002; ver Bandalos, Finney, y Geske, 2003) muestran que las estrategias metacognitivas junto con las estrategias de gestión de recursos emergen como buenos predictores del rendimiento, se esperaba que el control de acción y la esperanza de rasgo tuvieran un efecto indirecto en el logro académico en virtud de las estrategias metacognitivas y de gestión de recursos (Hipótesis 3). 


\section{Método}

\section{Participantes}

La muestra total consistía en 275 estudiantes de grado voluntarios (11 varones, 264 mujeres, edad media $=20,4$ años, $S d=2.8$, edades comprendidas entre 18-39 años), todos matriculados en la Facultad de Educación de las Universidades de Ioánnina y Tesalía, o en la Facultad de Psicología de la Universidad de Aristóteles de Tesalónica, Grecia.

\section{Instrumentos}

La Action Control Scale (ACS-90) [Escala de Control de Acción- ACS-90]. Para poder evaluar las diferencias en la orientación acción-estado, Kuhl (1985) desarrolló el instrumento autoinforme llamado la Action Control Scale, ACS (la versión alemana es la HAKEMP). Desde su elaboración original, la ACS ha pasado por tres revisiones, la versión más reciente es la ACS-90 (ver Diefendorff et al., 2000). La ACS-90 consiste en 36 ítems, divididos igualmente en tres subescalas que miden la orientación de acción en relación con el fracaso, con la decisión y con el rendimiento (Kuhl y Beckmann, 1994).

Los ítems de la escala describen breves escenarios que ocurren en la vida diaria y requieren elegir, entre dos opciones, la que indica lo que haría el participante. Una de las opciones indica una orientación a la acción y la otra una orientación al estado. Para puntuar los valores de la prueba, el constructor recomienda usar las respuestas orientadas a la acción. Se asigna una puntuación de uno a las respuestas que corresponden a la orientación a la acción y cero a las respuestas de orientación al estado. Se suman las puntuaciones para cada una de las tres subescalas y las puntuaciones más altas indican una mayor orientación a la acción. Algunos ejemplos de los ítems son los siguientes: para la Escala de Preocupación "Si acabo de comprar algún equipo (por ejemplo, un reproductor de DVD) y casualmente se cae al suelo y se daña de forma irreparable... : A. Me sobrepondría a esto rápidamente. B. Me llevaría mucho tiempo superarlo."; para la Escala de Vacilación "Cuando sé que debo terminar algo pronto...: A. Tengo que obligarme a empezar. B. Encuentro fácil ponerme a hacerlo y a terminarlo de una vez."; para la Escala de Volatilidad "Cuando leo algo que me parece interesante.... A. Aún así, a veces deseo dejar el artículo y hacer otra cosa. B. Me siento y leo el artículo durante un rato largo."

Trabajos previos empíricos y teóricos sugieren distintos modelos a priori de la estructura de factores de la ACS. El primero y más parsimonioso de estos modelos es el modelo de un solo factor, el cual concuerda con la idea de un constructo global y unitario de 
la orientación acción-estado. El segundo modelo, el modelo de dos factores, sugiere que hay dos constructos latentes que influyen en las respuestas de la ACS - la capacidad de escapar del modo de procesar orientado al estado (evaluada por los ítems de preocupación y vacilación) y la capacidad de permanecer en el modo de procesar orientado a la acción (evaluado por los ítems de volatilidad). El tercer modelo a priori, el modelo de tres factores, queda implícito en la mayoría de los trabajos previos empíricos y teóricos que emplean la medida ACS, y sugiere que la preocupación, la vacilación y la volatilidad son constructos separados.

En un intento por mejorar la ACS, Diefendorff et al. (2000) examinaron la estructura de factores de la ACS -90 realizando análisis factorial confirmatorio y comparando los tres modelos mencionados. Los primeros análisis factoriales confirmatorios de los tres modelos no mostraron buen ajuste de los datos. Se eliminaron los ítems que no funcionaron bien, y se probaron los tres modelos nuevamente, resultando en un apoyo a la solución de tres factores correspondientes con las subescalas de orientación a la acción en su relación con el fracaso (AOF), en su relación con la decisión (AOD), y con el rendimiento (AOP). La Action Control Scale revisada conservó 8 de los 12 ítems originales de la subescala de AOF, 8 de los 12 ítems originales de la subescala de AOD, y 6 de los 12 ítems originales de la subescala de AOP.

Para un trabajo previo, el primer autor había traducido la ACS-90 al griego y un psicólogo bilingüe independiente la volvió a traducir al idioma original (ver Papantoniou 2002; Papantoniou y Efklides, 2004). El cuestionario vuelto a traducir se comparó con el original y se le aplicaron unas pequeñas modificaciones.

Examinamos la estructura de factores de la versión griega de la Action Control Scale, usando análisis factorial confirmatorio ${ }^{1}$ para comparar las tres estructuras de factores a priori, implícitas en investigaciones previas teóricas y empíricas, pero ninguno de los modelos se ajusta realmente bien con los datos (Papantoniou, Moraitou, Dinou, y Katsadima, presentado a publicación). Por consiguiente, se llevó a cabo una segunda serie de análisis confirmatorios de los tres modelos, usando un grupo revisado de ítems de las tres subescalas ACS-90. En el grupo revisado de ítems, se excluyeron 14 ítems de los que propusieron Diefendorff et al. (2000), debido a su pobre funcionamento. Se excluyeron 4 ítems de la subescala AOF (ítems 1, 7, 16, y 25) y de la subescala AOD (ítems 14, 17, 23, y 32). Se eliminaron 6 ítems de la subescala AOP (ítems 6, 9, 12, 18, 27, y 30). Los modelos que empleaban el grupo revisado de ítems se ajustaban notablemente mejor con los datos que los del primer grupo de análisis. Por lo tanto, basándonos en las pruebas de diferencia del chi-cuadrado, comparaciones con el CFI

\footnotetext{
${ }^{1}$ Para todos los análisis factoriales confirmatorios se usó la EQS 6.1 (Benler, 2005).
} 
(Índice de Ajuste Comparativo), y el bajo valor de sus RMSEA (Error de Aproximación Cuadrático Medio) y SRMR (Residuo Cuadrático Medio Estandarizado), el modelo que mejor se ajustaba fue inequívocamente el modelo de tres factores con interrelaciones entre los factores latentes, $\chi^{2}(206, N=323)=259,90, p=.006, \chi^{2} / d f=1,26, \mathrm{CFI}=.921, \mathrm{SRMR}=.051$, RMSEA = .029 ( CI $_{90 \%}, 020$ a ,039) (Papantoniou et al., presentado presentado a publicación). Además, este modelo tiene también la ventaja de una validación teórica y empírica previa (ver Diefendorff et al., 2000).

Como se muestra en la Tabla 1, el análisis factorial confirmatorio verificó la estructura de tres factores de la ACS-90 revisada para esta muestra, a saber, la orientación de acción en relación con el Fracaso, en relación con la Decisión y en relación con el Rendimiento, $\chi^{2}(206$, $N=275)=277,7, p<, 001, \chi^{2} / d f=1,01, \mathrm{CFI}=, 894$ (marginal), SRMR $=, 055$, RMSEA $=$ ,036 ( $\mathrm{CI}_{90 \%}, 024$ a ,046) (ver Brown, 2006). Los valores del $\alpha$ Cronbach eran ,70 para la Escala de Preocupación, ,68 para la Escala de Vacilación, y ,58 para la Escala de Volatilidad. En trabajos previos, sea con la versión original o revisada en inglés (Diefendorff et al., 2000; Jaramillo y Spector, 2004; Kuhl, 1994b) o con la versión original en griego (Papantoniou, 2002), los coeficientes del $\alpha$ Cronbach, para las tres subescalas, oscilaban entre ,51 y,78. En este trabajo, al igual que en trabajos previos, la consistencia interna de las tres subescalas de la ACS-90 tiende a ser un tanto baja, probablemente porque los cálculos basados en alfa estén estimados a la baja, dado que los ítems son dicótomos y no reúnen las condiciones de tau equivalencia (Cortina, 1993, p.101).

Tabla 1.La estructura de la Action Control Scale revisada (solución estandarizada),

en la muestra de la prueba

\begin{tabular}{|c|c|c|c|c|c|}
\hline \multicolumn{6}{|c|}{ Factores } \\
\hline Ítems & $\begin{array}{l}\text { AOF } \\
(\mathrm{F} 1)\end{array}$ & $\begin{array}{l}\text { AOD } \\
\text { (F2) }\end{array}$ & $\begin{array}{l}\mathrm{AOP} \\
\text { (F3) }\end{array}$ & $E$ & $\underline{\mathrm{R}^{2}}$ \\
\hline $\mathrm{AOF} 4$ & 470 & & & 883 & 221 \\
\hline AOF10 &, 525 & & & 851 & 275 \\
\hline AOF13 & 371 & & & ,929 & 138 \\
\hline AOF19 & ,453 & & & ,891 & ,205 \\
\hline AOF22 &, 445 & & &, 896 & , 198 \\
\hline AOF28 &, 474 & & &, 880 & 225 \\
\hline AOF31 &, 591 & & & ,807 & ,349 \\
\hline AOF34 & ,468 & & &, 884 & 219 \\
\hline AOD2 & & ,375 & & ,927 & 140 \\
\hline AOD5 & &, 385 & & ,923 & 148 \\
\hline AOD8 & & ,440 & & ,898 & 194 \\
\hline AOD11 & &, 423 & & ,906 & 179 \\
\hline AOD20 & &, 565 & &, 825 & 319 \\
\hline AOD26 & &, 514 & &, 858 & ,264 \\
\hline AOD29 & &, 536 & &, 844 & ,287 \\
\hline AOD35 & & ,417 & & ,909 & 174 \\
\hline
\end{tabular}




\begin{tabular}{|c|c|c|c|c|}
\hline AOP3 & & , 198 & ,980 & 039 \\
\hline AOP15 & & 632 &, 775 & ,399 \\
\hline AOP21 & & 230 & 973 & 053 \\
\hline AOP24 & & ,474 &, 881 & 224 \\
\hline AOP33 & & 673 &, 739 & 453 \\
\hline AOP36 & & ,333 & ,943 &, 111 \\
\hline \multicolumn{5}{|l|}{ Correlaciones de factores } \\
\hline $\mathrm{F} 2(\mathrm{AOD})-\mathrm{F} 1(\mathrm{AOF})$ & ,495 & & & \\
\hline $\mathrm{F} 3(\mathrm{AOP})-\mathrm{F} 1(\mathrm{AOF})$ & 088 & & & \\
\hline $\mathrm{F} 3(\mathrm{AOP})-\mathrm{F} 2(\mathrm{AOD})$ &, 335 & & & \\
\hline
\end{tabular}

Nota: $\mathrm{AOF}=$ Factor de Orientación de Acción relacionado con el Fracaso; AOD $=$ Factor de Orientación de Acción relacionado con la Decisión; AOP = Factor de Orientación de Acción relacionado con el Rendimiento.

La Adult Dispositional Hope Scale (ADHS) [Escala de Disposición a la Esperanza en Adultos]. La ADHS de Snyder et al. (1991) es una escala de 12 ítems diseñada para explorar la disposición a la esperanza en adultos. Cuatro de estos ítems se refieren al pensamiento orientado a caminos y cuatro de los ítems al pensamiento orientado a la agencia. Un ejemplo para el pensamiento orientado a caminos sería "Hay muchas formas de salvar cualquier problema", y para el pensamiento orientado a la agencia "Mis experiencias pasadas me han preparado bien para el futuro". Finalmente, hay cuatro ítems de distracción (p.ej., "Me siento cansado la mayor parte del tiempo"). Los datos reunidos con estos cuatro últimos no se incluyeron en el análisis.

Para un trabajo previo, el segundo autor tradujo al griego la ADHS (Moraitou, Kolovou, Papasozomenou, y Paschoula, 2006). Los participantes debían contestar si cada uno de los 12 ítem eran cierto sobre ellos mismos, en una escala de 5 puntos tipo Likert, desde 1 (nada cierto) al 5 (muy cierto) y no en la escala de 8 puntos propuesta por Snyder et al. (1991; ver también Moraitou et al., 2006). El análisis factorial confirmatorio ratíficó la estructura de dos factores de la ADHS para esta muestra, o sea, factores de Waypower ('Pensamiento orientado a caminos') y Fuerza de Voluntad ("Pensamiento orientado a la Agencia"), $\chi^{2}(19, N$ $=275)=60,30, p<, 001, \chi^{2} / d f=3,44, \mathrm{CFI}=, 907, \mathrm{SRMR}=, 065, \mathrm{RMSEA}=, 089\left(\mathrm{CI}_{90 \%}, 064\right.$ a ,115), (ver Brown, 2006). La consistencia interna para los factores fue: $\alpha$ Cronbach $=, 74 \mathrm{y}$ , 61 , respectivamente.

El Motivated Strategies for Learning Questionnaire (MSLQ) [Cuestionario de Estrategias de Motivación para el aprendizaje]. El MSLQ lo elaboraron Pintrich et al. (1991) como una medida de autorregulación del aprendizaje. El MSLQ consta de dos secciones, la sección de motivación y la sección de estrategias de aprendizaje. En este trabajo se usó el 
apartado de estrategias de aprendizaje para evaluar el uso de estrategias de aprendizaje por parte de los alumnos universitarios. El apartado de estrategias de aprendizaje del MSLQ consta de 50 ítems, divididos en nueve subescalas para medir: el ensayo, la elaboración, la organización, y el pensamiento crítico (que representan el aspecto cognitivo de la autorregulación del aprendizaje); la metacognición (que representa el constructo metacognitivo de la autorregulación); y la gestión del entorno y del tiempo, la regulación del esfuerzo, aprendizaje entre iguales, y la búsqueda de ayuda (que representan el componente de gestión de la autorregulación). Las respuestas se hacen en una escala de 7 puntos tipo Likert, desde el 1 (nada cierto para mí) hasta el 7 (muy cierto para mí). Un ejemplo de la subescala usada para medir la elaboración es "Cuando leo para esta clase, intento relacionar el material con algo que ya conozco". Un ejemplo de la subescala usada para medir la metacognición es "Cuando estudio para esta clase, fijo metas a mí mismo para así dirigir mis actividades en cada período de estudio". Un ejemplo de la subescala usada para medir la gestión del entorno es "Normalmente estudio en un lugar donde puedo concentrarme en el trabajo del curso".

El instrumento MSLQ se ha usado ampliamente para investigar las estrategias de motivación y aprendizaje de los alumnos en muchos países, tales como Arabia, Australia, Canadá, China, Japón, y Taiwán (ver Chen, 2002). A efectos de este estudio, dos de los autores y una persona bilingüe independiente tradujeron al griego la sección sobre estrategias de aprendizaje del MSLQ. Se compararon las dos versiones del cuestionario traducido y la persona bilingüe sugirió modificaciones que se aplicaron a la versión del cuestionario traducida por los autores. El análisis factorial confirmatorio verificó la estructura de nueve factores de la sección de estrategias de aprendizaje del MSLQ para esta muestra, según algunos índices de ajuste que resultaron comparables con aquellos usados por Pintrich et al. (1991) para confirmar la estructura de nueve factores: $\chi^{2}(1139, N=275)=2635,4, p<, 001, \chi^{2} / d f=2,31$, LISREL GFI $=, 71, \mathrm{SRMR}=, 09$ (Pintrich et al., 1991: $\chi^{2} / d f=2,26$, LISREL GFI = ,78, SRMR = ,08). Basados en estos índices, Pintrich et al. (1991, p. 79-80) afirmaron: “Aunque las índices de bondad de ajuste no sean fantásticas, no obstante, son valores bastante razonables, dado el hecho de que estamos abarcando una amplia gama de cursos y materias. Actitudes de motivación y aplicaciòn de estrategias de aprendizaje pueden variar dependiendo de las características del curso, las exigencias del docente, y las características individuales del alumno. En general, los modelos muestran estructuras válidas, y se puede afirmar razonablemente la validez factorial de las escalas del MSLQ". 
En este trabajo, los estimados alfa Cronbach de consistencia interna son comparables con los obtenidos por Pintrich et al. (1991): $\alpha=, 66$ (Pintrich et al., 1991: $\alpha=, 69$ ) para el ensayo, $\alpha=, 71$ (Pintrich et al., 1991: $\alpha=$,76) para la elaboración, $\alpha=, 82$ (Pintrich et al., 1991: $\alpha=$,64) para la organización, $\alpha=, 67$ (Pintrich et al., 1991: $\alpha=, 80$ ) para el pensamiento crítico, $\alpha=, 59$ (Pintrich et al., 1991: $\alpha=, 79)$ para la metacognición, $\alpha=, 72$ (Pintrich et al., 1991: $\alpha=$,76) para la gestión del entorno y del tiempo, $\alpha=, 70$ (Pintrich et al., 1991: $\alpha=, 69$ ) para la regulación de esfuerzo, $\alpha=, 64$ (Pintrich et al., 1991: $\alpha=, 76$ ) para aprendizaje entre iguales, y $\alpha=, 53$ (Pintrich et al., 1991: $\alpha=, 52$ ) para la búsqueda de ayuda.

Logro académico. Se midió el logro académico con la nota final del alumno para la asignatura, la cual fue pasada a una escala de 10 puntos $(M=6,52 ; D t=2,33)$. Evaluación para la nota final constaba de: (a) una redacción de 3 puntos que suponía la producción de prosa original sobre temas asignados por el docente, y (b) una prueba de 7 puntos que se administró al final del semestre y que exigía recordar información de los libros de texto.

\section{Procedimiento}

Antes de comenzar este trabajo, se obtuvo permiso para llevar a cabo una investigación con sujetos humanos. Se administró el cuestionario a alumnos de cuatro asignaturas básicas de psicología (principalmente temas de psicología educativa, cognitiva y evolutiva). Aunque se trataba de dos profesores distintos para estas asignaturas (un alumno de post doctorado y un profesor titular), se usaron algunos libros de texto y deberes de clase similares en los cuatro cursos. Se aplicaron los cuestionarios en el aula. La ACS-90 y la ADHS fueron aplicadas a principios del semestre. Los participantes escribieron sus respuestas a los cuestionarios, que fueron presentados en orden aleatorio. También dieron información demográfica, incluidos la edad, el sexo y su curso antes de completar los cuestionarios. El MSLQ se administró durante una segunda sesión al final del semestre. La participación en el estudio fue voluntaria y se informó a los participantes que todos los resultados eran confidenciales.

\section{Análisis estadísticos}

Se procedió a usar el análisis del camino-una técnica de modelado de ecuaciones estructurales (SEM) para analizar los modelos estructurales con variables observadasviendo que es adecuado para examinar las relaciones entre múltiples constructos evaluados 
con escalas sumativas (Jaramillo y Spector, 2004), y que está ampliamente representado en la literatura sobre la autorregulación del aprendizaje. Se calculó un análisis del camino con variables manifiestas, específicamente para examinar las relaciones entre las dimensiones de la orientación a la acción, la disposición a la esperanza, las estrategias de autorregulación del aprendizaje y el logro académico. Debido al relativamente pequeño tamaño de la muestra, no se realizó un análisis al nivel de ítems. En su lugar, se basó la matriz de covarianza en los resultados globales para la separación, la iniciativa, la persistencia, el pensamiento orientado a caminos, el pensamiento orientado a la agencia, el ensayo, la elaboración, la organización, el pensamiento crítico, la metacognición, la gestión del entorno y del tiempo, la regulación del esfuerzo, el aprendizaje entre iguales, la búsqueda de ayuda y el logro académico. El rendimiento evaluado con notas académicas y las nueve estrategias de autorregulación del aprendizaje se definieron como variables endógenas. Las tres dimensiones de orientación a la acción, al igual que los dos componentes de la disposición a la esperanza, se definieron como variables exógenas.

Se llevó a cabo el análisis del camino con el programa EQS Versión 6.1 y se ejecutó la matriz de covarianza usando el procedimiento de estimación de Probabilidad Máxima (Bentler, 2005). Comenzando con la matriz de covarianza, se ejecutaron diversos modelos de estimaciones de Probabilidad Máxima. Inicialmente, en la parte estructural del modelo, se le permitió que las tres variables independientes de control de acción y las dos de la disposición a la esperanza, incorporadas en el modelo del camino, se correlacionaran con y predijeran las nueve variables latentes de las estrategias de autorregulación del aprendizaje y la variable dependiente del logro académico. A la vez, se le permitió que las variables latentes de las estrategias de autorregulación del aprendizaje se correlacionaran con y predijeran la variable dependiente del logro académico también. Varias iteraciones, las cuales incluían modificaciones sugeridas e indicadas por las pruebas de Lagrange Multiplier and Wald [Multiplicador de Lagrange y Wald], dieron como resultado el modelo final. Se usó la prueba Wald para probar la necesidad de esas regresiones y para sugerir un modelo más restrictivo.

La no significación estadística de la prueba $\chi^{2}$ indica que el modelo teórico implícito reproduce significativamente las relaciones varianza-covarianza en la matriz. Ya que esta prueba es sensible al tamaño de la muestra, se evaluó también el ajuste del modelo usando el error de aproximación cuadrático medio (RMSEA). El RMSEA prueba el ajuste del modelo a la matriz de covarianza de la población. Una regla general es que RMSEA $\leq, 05$ indica un ajuste aproximado cercano y que valores entre ,05 y ,08 sugieren un error de aproximación razonable (Kline, 2005). Se usó también el Comparative Fit Index (CFI) [Índice de Ajuste Comparativo] que es uno de los índices que evalúan el mejoramiento relativo en el ajuste del modelo de investigación comparado con el modelo de referencia. Una regla general del CFI es 
que los valores mayores de ,90 pueden indicar un ajuste razonablemente para el modelo de investigación (Kline, 2005). Además, el ajuste de modelo se evaluó usando el Residuo Cuadrático Medio Estandarizado (SRMR). El SRMR es una medida del residuo de correlación media absoluta, la diferencia global entre las correlaciones observadas y las predichas. Valores en el SRMR menores de ,10 se consideran favorables generalmente (Kline, 2005).

Con respecto a los requisitos de tamaño de la muestra, para las técnicas de SEM, se recomienda como regla general que haya al menos cinco observaciones por parámetro estimado (Hair, Anderson, Tatham, y Black, 1998). Se estimaron 15 parámetros para el modelo del camino. Por lo tanto, el tamaño de la muestra para el modelo del camino debía ser superior a 75. Por tanto, el tamaño de la muestra superó el nivel mínimo recomendado para llevar a cabo el análisis del camino.

\section{Resultados}

Análisis del camino. La parte estructural del modelo final se presenta en la Tabla 2 y las correlaciones entre las variables independientes se muestran en la Tabla 3. El ajuste global de este modelo fue bueno, $\chi^{2}(30, N=275)=33,05, p=, 321, \chi^{2} / d f=1,10$. Los resultados indican que el SRMR es ,044, y el RMSEA es ,019 ( $\mathrm{CI}_{90 \%}, 000$ a ,051), lo que sugiere un buen ajuste de los datos (ver Brown, 2006). También, el CFI (,997) superó el umbral de ,90, lo que indica un ajuste adecuado (ver Brown, 2006).

Tabla 2. Modelo del camino donde se presentan relaciones entre control de acción, disposición a la esperanza, estrategias de autorregulación de aprendizaje y logro académico

\begin{tabular}{|c|c|c|c|c|c|c|c|c|c|}
\hline \multicolumn{10}{|c|}{ Variables independientes } \\
\hline $\begin{array}{l}\text { Variables } \\
\text { Dependientes }\end{array}$ & $\mathrm{AOF}$ & $\mathrm{AOD}$ & $\mathrm{AOP}$ & $\begin{array}{l}\mathrm{HO} \\
\mathrm{WI}\end{array}$ & $\begin{array}{c}\mathrm{HO} \\
\text { WAY }\end{array}$ & Metac & $\begin{array}{c}\text { TSE } \\
\mathrm{M}\end{array}$ & $\mathrm{E}$ & $\underline{\mathrm{R}^{2}}$ \\
\hline Ensayo & -.171 & & & .287 & & & & .950 & .098 \\
\hline Elaboración & & & & .244 & 107 & & & .951 & .095 \\
\hline Organización & & & & .229 & & & & .973 & .053 \\
\hline Pensamiento & & & & .170 & .123 & & & .968 & .064 \\
\hline Crítico & & & & & & & & & \\
\hline Metacognición & & .126 & & .269 & & & & .941 & .114 \\
\hline Gestión TEE & -.141 & .258 & & .332 & -.092 & & & .897 & .195 \\
\hline $\begin{array}{l}\text { Regulación de } \\
\text { Esfuerzo }\end{array}$ & -.147 & .205 & & .286 & & & & .917 & .158 \\
\hline $\begin{array}{l}\text { Logro } \\
\text { Académico }\end{array}$ & & & -.171 & & -.141 & .139 & .287 & .915 & .163 \\
\hline
\end{tabular}

$\mathrm{AOF}=$ Variable de Orientación a la Acción relacionada con el Fracaso; AOD = Variable de Orientación a la Acción relacionada con la Decisión; AOP $=$ Variable de Orientación a la Acción relacionada con el Rendimiento; HOWI = Variable de Fuerza de Voluntad (Esperanza); HOWAY = Variable de Waypower (Esperanza) -- Sólo se presentan en la tabla los efectos significativos de variables independientes. 
Como se planteó en las hipótesis $\mathrm{H} 1$ y H3, encontramos que dos dimensiones de orientación a la acción- la separación y la iniciativa- estaban relacionadas con el uso de algunas estrategias cognitivas, metacognitivas y autorreguladoras. En específico, la iniciativa estaba relacionada positivamente con las estrategias metacognitiva, la gestión de tiempo y del entorno de estudio y la regulación de esfuerzo. Parece que la capacidad del alumno para iniciar acciones previstas explica el uso de la estrategia metacognitiva y la de gestión del tiempo y entorno de estudio, lo cual se traduce en un mayor rendimiento evaluado con nota académica. Se encontró que la separación estaba relacionada negativamente con el ensayo, la gestión del tiempo y del entorno de estudio y la regulación del esfuerzo. Ya que la gestión del tiempo y del entorno de estudio se relaciona positivamente con el logro académico, tanto la capacidad del alumno de detener pensamientos sobre metas alternativas o eventos no deseados que interfieran en el progreso de la tarea en mano, como la capacidad de no estancarse en un estado inicial de logro de meta, pueden inhibir el logro a través de la regulación inapropiada de la gestión del tiempo y del entorno de estudio.

\section{Tabla 3. Correlaciones entre variables independientes del modelo del camino} presentadas en la Tabla 2

\begin{tabular}{lc}
\hline & Correlaciones entre las \\
HOWI - HOWAY &, 466 \\
AOF - HOWAY &, 217 \\
AOD - HOWAY &, 323 \\
AOP - HOWAY &, 153 \\
AOF - HOWI &, 140 \\
AOD - HOWI &, 387 \\
AOP - HOWI &, 276 \\
AOD - AOF &, 337 \\
AOP - AOD &, 237 \\
Elaboración - Ensayo &, 487 \\
Organización - Ensayo &, 666 \\
Pensamiento Crítico - Ensayo &, 173 \\
Metacognición - Ensayo &, 498 \\
Gestión TSE - Ensayo &, 522 \\
Regulación de Esfuerzo - Ensayo &, 315 \\
Organización - Elaboración &, 540 \\
Pensamiento Crítico - Elaboración &, 467 \\
Metacognición - Elaboración &, 564 \\
Gestión TSE - Elaboración &, 257 \\
Regulación de Esfuerzo - Elaboración &, 236 \\
Pensamiento Crítico - Organización &, 268 \\
Metacognición - Organización &, 481 \\
Gestión TSE - Organización &, 380 \\
Regulación de Esfuerzo - Organización &, 242 \\
Metacognición - Pensamiento Crítico &, 403 \\
Gestión TSE - Metacognición &, 427 \\
Regulación de Esfuerzo - Metacognición &, 386 \\
Regulación de Esfuerzo - Gestión TEE &, 555 \\
\hline
\end{tabular}


AOF $=$ Variable de Orientación a la Acción relacionada con el Fracaso; AOD = Variable de Orientación a la Acción relacionada con la Decisión; AOP = Variable de Orientación a la Acción relacionada con el Rendimiento; HOWI = Variable de Fuerza de Voluntad (Esperanza); HOWAY = Variable de Waypower (Esperanza) -- Sólo se presentan en la tabla los efectos significativos de variables independientes.

Sin embargo, contrario a la $\mathrm{H} 3$, encontramos que la persistencia está directa y negativamente relacionada con el rendimiento evaluado por notas académicas. Inesperadamente, parece ser que la capacidad del alumno de permanecer centrado en actividades relacionadas con una meta hasta finalizarlas, se traduce en un logro académico más bajo durante un examen rutinario, típico y bastante fácil de una asignatura introductoria de psicología. Otro hallazgo inesperado, en contraste con la H1a, fue la falta de relación entre la persistencia y las estrategias de autorregulación del aprendizaje.

Como se planteó en la $\mathrm{H} 2$ y la $\mathrm{H} 3$, encontramos que los dos componentes de la disposición a la esperanza, el pensamiento orientado a la agencia y el pensamiento orientado a caminos, estaban relacionados con el uso de varias estrategias cognitivas, metacognitivas y autorreguladoras. En específico, el pensamiento orientado a la agencia estaba positivamente relacionado con el uso de todas las estrategias cognitivas, metacognitivas y autorreguladoras, excepto las del aprendizaje entre iguales y la búsqueda de ayuda. Ya que la metacognición y la gestión del tiempo y del entorno de estudio están relacionadas positivamente con el logro académico, la capacidad percibida del alumno para ratificar un diálogo interno de agencia a la hora de encontrar impedimentos facilita el logro académico a través de la regulación eficaz del uso de la estrategias metacognitiva y la gestión de su tiempo y entorno de estudio.

Se encontró que el pensamiento orientado a caminos está positivamente relacionado con las estrategias cognitivas de la elaboración y el pensamiento crítico. Sin embargo, también se encontró que inhibe el logro académico a través de la regulación inapropiada de la gestión del tiempo y del entorno de estudio. Finalmente, contrario a la H3, se encontró que el pensamiento orientado a caminos está relacionado negativamente con el logro académico. Esto indica que la capacidad percibida del alumno de producir estrategias cognitivas alternativas hacia metas valiosas y de tener un diálogo interno positivo sobre su capacidad de encontrar estas estrategias impiden el rendimiento evaluado por nota académica, sea directa o indirectamente, a través de la regulación inapropiada del uso de la estrategia de gestión de recursos.

\section{Discusión}

El objetivo principal de este trabajo era examinar las relaciones entre el control de acción y la disposición a la esperanza por un lado, y el uso de estrategias de aprendizaje y el logro académico por otro lado. 
En cuanto a la eficacia predictora del control de acción y de la disposición a la esperanza en las estrategias de autorregulación del aprendizaje, el pensamiento orientado a la agencia se destaca de otros constructos. En específico, se encontró que el pensamiento orientado a la agencia afecta positivamente el uso de ensayo, elaboración, organización, pensamiento crítico, metacognición, gestión del tiempo y del entorno de estudio, y regulación del esfuerzo. Esto está acorde con su definición como "percepciones de los individuos respecto a sus capacidades de claramente conceptualizar metas y de iniciar y sostener la motivación necesaria para usar las estrategias específicas para alcanzar esas metas" (Snyder et al., 2002). Este hallazgo puede explicarse también tomando en consideración la similitud compartida entre el componente de agencia (fuerza de voluntad) de la esperanza, y la autoeficacia, ya que las creencias autoeficaces son fuertes predoctores de resultados educativos y han mostrado correlaciones con los procesos autorreguladores (Bandura, 1997).

El pensamiento orientado a caminos parece influir positivamente en algunas destrezas de aprendizaje de orden mayor, tales como la elaboración y el pensamiento crítico. Este hallazgo podría explicarse en base a la naturaleza compleja de las estrategias antes mencionadas que se asocia con la capacidad personal de ver un problema desde múltiples perspectivas. Sin embargo, el waypower como la búsqueda elaboradora de estrategias cognitivas alternativas parece dificultar el rendimiento, sea directa o indirectamente, a través de la regulación inapropiada del uso de la estrategia de gestión de recursos y por su naturaleza de "llevar más tiempo" y de ser más "creativa" que "ejecutiva".

En general, se obtienen varios resultados interesantes de este trabajo en relación con las tres dimensiones del control de acción. En específico, se encontró que la iniciativa afecta positivamente el uso de la metacognición, la gestión del tiempo y del entorno de estudio, y la regulación del esfuerzo. Este hallazgo es consecuente con investigaciones previas (Bembenutty et al., 1998; Jaramillo y Spector, 2004; Papantoniou, 2002; Roy, Vezeau, y Bouffard, 2008) que indican que, debido a su mayor capacidad para finalizar las cosas, los individuos orientados a la acción en relación con la decisión están dispuestos a supervisar la eficacia de su aprendizaje, a gestionar eficazmente su tiempo y entorno de estudio, y a ejercer un mayor esfuerzo en capacidades de aprendizaje, a pesar de posibles distracciones.

Contrario a la orientación a la acción relacionada con la decisión, la orientación relacionada con el fracaso parece bloquear el ensayo, la gestión del tiempo y del entorno de estudio y la regulación del esfuerzo. Parece que la rápida separación del fracaso puede llevar a la persona a eludir la búsqueda de causas, la cual aportaría a la autorregulación eficaz. Ya que la gestión de tiempo y del entorno de estudio está relacionada positivamente con el logro académico, este hallazgo es consecuente con la literatura que muestra que preocupación con 
el fracaso puede mejorar el rendimiento (Diefendorff, 2004; Kuhl y Fuhrmann, 1998; Perry et al., 2001). Otro hallazgo que confirma que la orientación al estado no es siempre disfuncional fue el efecto negativo de la persistencia en el logro académico. La investigación indica que los individuos orientados al estado funcionan de forma óptima cuando se les permite suficiente tiempo y se les libera de la presión, y cuando reciben instrucciones explícitas acerca de las actividades que deben completar. Aunque estas condiciones no se cumplen normalmente durante el transcurso típico de un examen, estos hallazgos indican que los estudiantes implicados en este trabajo experimentaron un ambiente amistoso en el examen y una orientación cercana. En resumen, los resultados de este trabajo tienden a reforzar el patrón variado de efectos del control de acción en el uso de estrategias de aprendizaje y en el rendimiento.

En resumen, se encontró que el control de la acción y la disposición a la esperanza, como constructos de volición y de motivación respectivamente, relacionados con las metas, estaban asociados con el uso de todas las estrategias cognitivas, metacognitivas y autorreguladoras, excepto la de aprendizaje entre iguales y la búsqueda de ayuda. Es posible que el aprendizaje entre iguales y la búsqueda de ayuda sean distintas de otras estrategias de aprendizaje en cuanto a que también son interacciones sociales. Por lo tanto, es posible que los motivos sociales influyan en el uso de esas estrategias.

Además, el trabajo reveló que las estrategias de autorregulación del aprendizaje, particularmente la metacognición y la gestión del tiempo y del entorno de estudio, mediaron la relación entre la separación, la iniciativa, el pensamiento orientado a caminos y el pensamiento orientado a la agencia, por un lado, y el logro académico por otro lado. Estos hallazgos son consecuentes con investigaciones previas que indican que la metacognición y la gestión del tiempo y del entorno de estudio son dos destacados predictores de logro académico (Bidjerano y Dai, 2007; Chen, 2002; ver Bandalos, Finney, y Geske, 2003). En específico, estos resultados sugieren que los alumnos que tienden a supervisar la eficacia de su aprendizaje y que aprenden a gestionar eficazmente su tiempo y su entorno de estudio, tienen mayor posibilidad de mejor rendimiento en una asignatura introductoria de psicología, frente a sus compañeros que no tienen estas cualidades. Tomando en cuenta también los efectos negativos de la persistencia y del pensamiento orientado a caminos sobre el rendimiento evaluado por notas académicas, parece ser que los alumnos que se describen a sí mismos como pobres en persistencia y en el pensamiento orientado a caminos tienen más probabilidades de éxito en el logro académico.

Finalmente, un hallazgo interesante es la asociación entre el control de acción y la disposición a la esperanza. Ya que ambos son constructos dirigidos a metas, una explicación 
convincente para esta relación puede ser el hecho de que los individuos orientados a la acción en su relación con el fracaso, con la decisión y con el rendimiento son capaces de separarse de pensamientos sobre metas alternativas que pueden interferir en el progreso de la tarea en mano, de iniciar fácilmente el trabajo de la tarea y de eficazmente centrarse en la intención hasta finalizar la tarea. Esto se debe a su capacidad de dirigir la motivación necesaria por el mejor camino a través del pensamiento orientado a la agencia y del diálogo interno y de producir estrategias alternativas con el objetivo de finalizar la tarea.

El trabajo presente ofrece algunas de las características previas de los alumnos y su potencial de impacto en el aprendizaje, de lo cual deben estar conscientes los docentes. En específico, este trabajo encontró que las estrategias de autorregulación del aprendizaje y del logro académico tienen correlaciones importantes con las características de rasgo, tales como el control de acción y la disposición a la esperanza. Sin descontar el supuesto de que la autorregulación del aprendizaje, en general, es una característica que se puede aprender, los resultados de este trabajo sugieren que los docentes deben estar conscientes de las predisposiciones de personalidad que cada alumno trae a cada situación específica de aprendizaje (ver Bidjerano y Dai, 2007). Desde un punto de vista de intervención, la evaluación formal e informal del control de acción y la disposición a la esperanza puede indicar al instructor las personas que desarrollarán de forma natural las destrezas de autorregulación y las que quizás no las desarrolle sin un entrenamiento explícito. Como consecuencia, este conocimiento sobre la relación entre estos dos constructos relacionados con la meta y la autorregulación del aprendizaje permitirá que los docentes estén mejor preparados para diseñar intervenciones individualizadas según corresponde.

Una limitación de este trabajo es el uso de medidas de autoinforme de las capacidades de autorregulación. Hacen falta diseños más rigurosos para establecer la validez de la relación entre la autorregulación académica y las características de rasgo, usando medidas del comportamiento y de observación de la autorregulación (es decir, medidas en tiempo real de las estrategias de aprendizaje o evaluación de estrategias usando grabaciones de vídeo) (ver Dermitzaki, 2004). Se debe reconocer la naturaleza reducida de la muestra, en especial al considerar la edad y el sexo. Se desconoce también si se habría obtenido el mismo patrón de resultados si se hubiese incluido alumnos de otras Facultades, aparte de la Facultad de Educación. Futuros trabajos, con otros grupos de universitarios y con grupos de diferentes edades, deberían aclarar aún más cómo el control de acción y la esperanza predisponen a los individuos a emplear la autorregulación del aprendizaje, y cómo estas disposiciones intervienen y interactúan con condiciones de aprendizaje en el desarrollo de estrategias autorreguladoras. 
En conclusión, la intención de este trabajo era llamar la atención a algunas conexiones profundas entre la autorregulación del aprendizaje, el control de acción y la disposición a la esperanza, y no llegar a respuestas definitivas. Por lo tanto, la mayor contribución de este trabajo es la identificación de características de tipo rasgo, tales como el control de acción y la disposición a la esperanza, como fuente de la autorregulación del aprendizaje en alumnos universitarios.

\section{Referencias}

Bandalos, D. L., Finney, S. J., y Geske, J. A. (2003). A model of statistics performance based on achievement goal theory. Journal of Educational Psychology, 95(3), 604-616.

Bandura, A. (1997). Self-efficacy: The exercise of control. Nueva York: Freeman.

Bartels, J. M., Magun-Jackson, S., y Kemp, A. (2009). Volitional regulation and selfregulated learning: An examination of individual differences in approach-avoidance achievement motivation. Electronic Journal of Research in Educational Psychology, $7(2), 605-626$.

Bembenutty, H., Karabenick, S. A., McKeachie, W. J., y Lin, Y-G. (Abril, 1998). Academic delay of gratification as a volitional strategy. Trabajo presentado en la Reunión Anual de la Asociación Americana de Investigación Educativa, San Diego, CA.

Bentler, P. M. (2005). EQS 6.1. Encino, CA: Multivariate Software, Inc.

Bidjerano, T., y Dai, D. Y. (2007). The relationship between the big-five model of personality and self-regulated learning strategies. Learning and Individual Differences, 17, 69-81.

Boekaerts, M. (1997). Self-regulated learning: A new concept embraced by researchers, policy makers, educators, teachers, and students. Learning and Instruction, 7, 161186.

Brown, T. A. (2006). Confirmatory factor analysis for applied research. Nueva York: Guilford.

Brunstein, J. C., y Olbrich, E. (1985). Personal helplessness and action control: Analysis of achievement-related cognitions, self assessments, and performance. Journal of Personality and Social Psychology, 48, 1540-1551.

Chen, C. S. (2002). Self-regulated learning strategies and achievement in an introduction to information systems course. Information Technology, Learning, and Performance Journal, 20(1), 11-25.

Cortina, J. M. (1993). What is coefficient alpha? An examination of theory and applications. Journal of Applied Psychology, 78, 98-104. 
Curry, L. A., Snyder, C. R., Cook, D. L., Ruby, B. C., y Rehm, M. (1997). Role of hope in academic and sport achievement. Journal of Personality and Social Psychology, 73, 1257-1267.

Danner, D., Snowdon, D., y Friesen, W. (2001). Positive emotions in early life and longevity: Findings from the Nun study. Journal of Personality and Social Psychology, 80(5), 804-813.

Dermitzaki, I. (2004). Introduction: Individual and social processes in the regulation of learning. Hellenic Journal of Psychology, 1(2), 119-127.

Diefendorff, J. M. (2004). Examination of the roles of action-state orientation and goal orientation in the goal-setting and performance process. Human Performance, 17(4), 375-395.

Diefendorff, J. M., Hall, R. J., Lord, R. G., y Strean, M. L. (2000). Action-state orientation: Construct validity of a revised measure and its relationship to work-related variables. Journal of Applied Psychology, 85, 250-263.

Efklides, A., Niemivirta, M., y Yamauchi, H. (2002). Introduction: Some issues on selfregulation to consider. Psychologia: An International Journal of Psychology in the Orient, 45, 207-210.

Hair, J., Anderson, R., Tatham, R., y Black, W. (1998). Multivariate data analysis. NJ: Prentice Hall.

Hong, E., y O’Neil, H. F. (2001). Construct validation of a trait self-regulation model. International Journal of Psychology, 36(3), 186-194.

Jaramillo, F., y Spector, P. E. (2004). The effect of action orientation on the academic performance of undergraduate marketing majors. Journal of Marketing Education, 26, 250-260.

Kashdan, T., Pelham, W., Lang, A., Hoza, B., Jacob, R., Jennings, R., Blumenthal, J., y Gnagy, E. (2002). Hope and optimism as human strengths in parents of children with externalizing disorders: Stress in the eye of the beholder. Journal of Social and Clinical Psychology, 21(4), 441-468.

Kline, R. (2005). Principles and practice of structural equation modeling. Nueva York: The Guilford Press.

Kuhl, J. (1985). Volitional mediators of cognition-behavior consistency: Self-regulatory processes and action versus state orientation. In J. Kuhl \& J. Beckmann (Eds.), Action control: From cognition to behavior (pp. 101-128). Nueva York: Springer Verlag. 
Kuhl, J. (1994a). Action versus state orientation: Psychometric properties of the action control scale (ACS-90). In J. Kuhl \& J. Beckmann (Eds.), Volition and personality: Action versus state orientation (pp. 47-59). Seattle, WA: Hogrefe \& Huber.

Kuhl, J. (1994b). A theory of action and state orientations. In J. Kuhl \& J. Beckmann (Eds.), Volition and personality: Action versus state orientation (pp. 9-46). Seattle, WA: Hogrefe \& Huber.

Kuhl, J., y Beckmann, J. (1994). Volition and personality: Action versus state orientation. Seattle, WA: Hogrefe \& Huber.

Kuhl, J., y Fuhrmann, A. (1998). Decomposing self-regulation and self-control: The Volitional Components Inventory. In: J. Heckhausen \& C. S. Dweck (Eds.), Motivation and self-regulation across the life span (pp.15-49). Nueva York: Cambridge University Press.

Moraitou, D., Kolovou, C., Papasozomenou, C., y Paschoula, C. (2006). Hope and adaptation to old age: Their relationship with individual-demographic factors. Social Indicators Research, 76, 71-93.

Papantoniou, G. (2002). Cognitive style, anxiety and action control. Tesis no publicada, Universidad Aristóteles de Tesalónica (en griego).

Papantoniou, G., y Efklides, A. (2004). Affective and cognitive effects on action control. Psychology, 11, 285-302 (en griego).

Papantoniou, G., Moraitou, D., Dinou, M., y Katsadima, E. (presentado). Psychometric properties of the Greek version of the Action Control Scale. The International Journal of Educational and Psychological Assessment.

Perry, R. P., Hladkyj, S., Pekrun, R. H., y Pelletier, S. T. (2001). Academic control and action control in the achievement of college students: A longitudinal field study. Journal of Educational Psychology, 93(4), 776-789.

Peterson, S. J., Gerhardt, M. W., y Rode, J. C. (2006). Hope, learning goals, and task performance. Personality and Individual Differences, 40, 1099-1109.

Pintrich, P. R. (1995). Understanding self-regulated learning. In P. R. Pintrich (Ed.), Understanding self-regulated learning (pp. 3-12). San Francisco, CA: Jossey-Bass.

Pintrich, P. R. (1999). The role of motivation in promoting and sustaining self-regulated learning. International Journal of Educational Research, 31, 459-470.

Pintrich, P. R. (2000). The role of goal orientation in self-regulated learning. In M. Boekaerts, P. R. Pintrich, \& M. Zeidner (Eds.), Handbook of self-regulation (pp. 432-502). San Diego: Academic Press. 
Pintrich, P. R., y DeGroot, E. (1990). Motivational and self-regulated learning components of classroom academic performance. Journal of Educational Psychology, 82, 33-40.

Pintrich, P. R., Smith, D. A. F., Garcia, T., y McKeachie, W. J. (1991). A manual for the use of the Motivated Strategies for Learning Questionnaire (MSLQ). Ann Arbor: Universidad de Michigan.

Roy, M., Vezeau, C., y Bouffard, T. (May, 2008). Action orientation and self-regulation in school. Cartel presentado en la $3^{\text {a }}$ Reunión Bienal del Grupo de Interés Especial EARLI, 16 METACOGNITION, Ioánnina, Grecia.

Schunk, D. H., y Zimmerman, B. J. (Eds.). (1994). Self-regulation of learning and performance. Hillsdale, NJ: Erlbaum.

Snyder, C. R. (2000). Handbook of hope: Theory, measures, and applications. San Diego CA: Academic.

Snyder, C. R., Rand, K. L., y Sigmon, D. R. (2002). Hope theory: A member of the positive psychology family. In C. R. Snyder \& S. J. Lopez (Eds.), Handbook of positive psychology (pp. 257-275). Nueva York: Oxford University Press.

Snyder, C. R., Harris, C., Anderson, J. R., Holleran, S. A., Irving, L. M., Sigmon, S. T., Yoshinobu, L., Gibb, J., Langelle, C., y Harney, P. (1991). The will and the ways: Development and validation of an individual-differences measure of hope. Journal of Personality and Social Psychology, 60, 570-585.

Torrano, F., y Gonzalez, M. C. (2004). Self-regulated learning: Current and future directions. Electronic Journal of Research in Educational Psychology, 2(1), 1-34.

Zimmerman, B. J. (1999). Commentary: Toward a cyclically interactive view of self-regulated learning. International Journal of Educational Research, 31, 545-551. 